\title{
Optimizing Patient Management in Crohn's Disease in a Tertiary Referral Center: the Impact of Fast-Track MRI on Patient Management and Outcomes
}

\author{
Akos Ilias $^{1 *}$, Barbara D. Lovasz ${ }^{1,2^{*}}$, Lorant Gonczi ${ }^{1}$, Zsuzsanna Kurti ${ }^{1}, Z_{\text {Zsuzsanna Vegh }}{ }^{1}$, Liza D. Sumegi $^{1}$, Petra A. Golovics ${ }^{3}$, \\ Gabor Rudas ${ }^{4}$, Peter L. Lakatos ${ }^{1,5}$
}

1) Semmelweis University,

First Department of Medicine,

Budapest, Hungary

2) Institute of Applied

Health Sciences, Semmelweis

University, Budapest, Hungary

3) Military Hospital - State

Health Centre, Budapest,

Hungary

4) Semmelweis University,

Magnetic Resonance Imaging

Research Center, Budapest,

Hungary

5) Division of

Gastroenterology, McGill

University Health Centre,

Montreal, Quebec, Canada

\section{ABSTRACT}

Background \& Aims: Rapid optimization of treatment algorithms and disease outcomes requires an objective measurement of disease activity in patients with Crohn's disease (CD). Our aim was to evaluate the impact of rapid-access to magnetic resonance imaging (MRI) on treatment optimization, clinical decision-making and outcomes for CD patients in a specialized tertiary care for inflammatory bowel disease (IBD) patients. Methods: A cohort of 75 referral CD patients (median age: 34, IQR: 25-43 years) who had underwent 90 fasttrack MR enterography (MRE) scans between January 2014 and June 2016 were retrospectively enrolled. The MRI results were compared to clinical activity scores and biomarkers (C-reactive protein). The immediate impact of fast-track MRI on clinical decision-making, including changes in medical therapy, the need of hospitalization and surgery were evaluated.

Results: The location of CD was ileo-colonic in $61 \%$ of the patients with perianal fistulas in $56 \%$ and previous surgeries in 55\%. The indication for fast-track MRI scans was active disease (clinical or biomarker activity) in 55.6\%. The radiological activity (including mild radiological signs to severe lesions) was detected in $94 \%$ of cases. Significant/severe MRI activity was depicted in $68 \%$ of these patients. Correlation between MRI radiological activity and clinical disease activity or colonoscopy was moderate (kappa: 0.609 and 0.652). A change in therapeutic strategy was made in $94.1 \%$ of cases with severe MRI radiological activity vs. $50 \%$ of patients without severe MRI radiological activity $(\mathrm{p}=0.001)$. Significant/severe MRI activity was followed by higher surgery rates among patients with clinical disease activity ( $50 \%$ vs. $12.5 \%$; $\mathrm{p}=0.013$ ). MRI performed on patients with clinical and biomarker remission identified disease activity in a significantly smaller proportion. Conclusions: Fast-track MRI had a great impact on patient management in CD patients with clinical or biomarker activity, leading to better patient stratification and faster optimization of the therapy (medical or surgical), while MRI revealed previously undiagnosed disease activity only in a small proportion of patients in clinical remission.

Key words: inflammatory bowel disease - magnetic resonance imaging - clinical activity - clinical outcome.

Abbreviations: CD: Crohn's disease; CDAI: Crohn's Disease Activity Index; CDEIS: Crohn's Disease Endoscopic Index of Severity; CRP: C-reactive protein; CT: computer tomography; IBD: inflammatory bowel diseases; MRE: magnetic resonance enterography; MRI: Magnetic resonance imaging; US: ultrasonography.

\section{INTRODUCTION}

The management of inflammatory bowel diseases (IBD) requires a complex therapeutic strategy with objective and continuous evaluation and monitoring of disease progress both at the time of diagnosis and during the course of the disease.

The therapeutic goals and patient monitoring have changed completely in recent years. This paradigm shift has been initiated by the evidence that targeting symptom-based outcome parameters may not largely alter the natural course of IBD [1]. Recently, the 'treat to target' strategy that uses objective clinical and biochemical outcome measures to modify therapy has been gaining popularity. A recent consensus on 'Selecting Therapeutic Targets in Inflammatory Bowel Disease' in IBD (STRIDE) [2] suggested that disease activity should be monitored objectively by endoscopy and clinical/patientreported outcome (PRO) parameters with biomarkers as adjunct measures serving as primary targets for the therapy [2]. In addition, objective reassessment of patients in a timely 
fashion was suggested. While resolution of lesions on crosssectional imaging is not considered to be a universal target, imaging techniques do have a complementary role in patients with Crohn's disease (CD) who are not adequately assessed by colonoscopy and they remain the gold standard in identifying extraluminal manifestations of CD $[2,3]$.

The recent ECCO guidelines concluded that cross-sectional imaging techniques (magnetic resonance imaging /magnetic resonance enterography - MRI/MRE, computed tomography - CT, enterography and transabdominal ultrasonography US) are complementary to endoscopy with an overall similar diagnostic accuracy higher than $80 \%$ [3-5]. A further advantage of MRI over CT is that MRI is characterized by a high soft tissue contrast, without the exposure to ionizing radiation, which makes this modality the ideal choice for detecting lesions in CD [3]. According to published data, MRI has equal or even superior diagnostic accuracy compared to ileocolonoscopy (in selected CD patients, e.g. stricturing and penetrating complications, etc.), and may substantially impact therapeutic decisions $[3,5,6]$. Transabdominal US was reported to have comparable overall sensitivity and specificity (85\%/98\%) to MRI and CT modalities in diagnosing ileal CD; however, in assessing colonic lesions and fistulas or intra-abdominal abscesses, US provides a slightly decreased diagnostic accuracy $[3,5,7]$. A recent study showed that clinically-based point of care US could play a significant role in guiding the therapeutic management in $\mathrm{CD}$, although the proper use of US required high expertise and training in characterizing certain lesions in $\mathrm{CD}$ [8].

Several studies aimed to evaluate the accuracy of MRI in assessing disease activity in $\mathrm{CD}$, however data on the value of routine, rapid-access MRI in the everyday clinical decisionmaking are few. Objective patient evaluation and monitoring became standard in many IBD centers, including regular assessment by biomarkers, endoscopy and cross-sectional imaging. However, due to limited access, the routine use of 'semi-urgent' MRI is infrequent [9]. Therefore, the present study aim was to evaluate the impact of fast-track MRI on treatment optimization, clinical decision-making and outcomes in a specialized tertiary care IBD center.

\section{METHODS}

Consecutive IBD patients who had underwent fast-track MRI between January 2014 and June 2016 in a single tertiary referral IBD center were included. Magnetic resonance imaging studies were performed to evaluate disease activity in patients with or without disease flares. All examinations were performed as 'rapid-access' procedures with a maximum waiting time of two weeks. Clinical data were collected and comprehensively reviewed. Disease location and behavior were classified according to Montreal classification. The Crohn's Disease Activity Index (CDAI) was used for the clinical activity score [10]. Clinical remission was defined as $\mathrm{CDAI}<150$ points or no fistula drainage as assessed by the Fistula Drainage Assessment in CD. Biochemical activity was evaluated by measuring serum $\mathrm{C}$-reactive protein (CRP) and albumin. Biomarker activity was defined as CRP levels $>10 \mathrm{mg} / \mathrm{L}$. Clinically active disease was defined based on the combination of the clinicians' evaluation,
CDAI score and laboratory parameters. Available colonoscopy and US/CT results within three months after MRI were also collected. Of note, objective patient evaluation and monitoring is applied in our center at referral and during follow-up, including routine, serial use of clinical scores and biomarkers as well as endoscopy and/or cross-sectional imaging methods (including all modalities, US, CT and MRI) [11].

Magnetic resonance imaging scans were carried out by a 3.0T MR unit (Philips Achieva and Insignia) at the Magnetic Resonance Imaging Research Center of Semmelweis University, Budapest. All scans were MRE procedures, being performed in prone position with breath-hold technique. An oral contrast agent (polyethylene glycol or methylcellulose solutions, 1500$2000 \mathrm{~mL} 30$ minutes before the examination) and $0.2 \mathrm{~mL} /$ $\mathrm{kg}$ body weight of intravenous gadolinium chelate contrast material was administered in all cases. Coronal and axial singleshot T2-weighted turbo spin echo, axial 3D diffusion weighted whole-body imaging with background body signal suppression (DWIBS) sequences were performed for the abdomen. Sagittal, coronal T2, axial T2 fat suppression (fs), axial 3D DWIBS and axial 3D Wave ( $\mathrm{T} 1$ native and post contrast $\mathrm{fs}$ ) sequences were used for the pelvis. 16-channel torso and multi coils were used.

One expert radiologist with extensive knowledge in the treatment of IBD patients evaluated the MRI scans. The MRI variables evaluated included bowel wall thickening, relative contrast enhancement (RCE, i.e. enhancement of the bowel wall after administration of intravenous gadolinium contrast material), presence of mucosal ulcers, mural edema (hyperintensity on T2-wedged sequences of the colon wall relative to the psoas muscle), enlarged regional lymph nodes (10 $\mathrm{mm})$, peri-enteric vascularization (comb sign), peri-enteric fluid or fat stranding, presence of abscesses or fistulas. Diffusion weighted imaging sequences were also performed, diffusion restriction being evaluated by an expert radiologist, without calculation of the value for apparent diffusion coefficient (ADC). Disease activity on the MRI scan was considered when more than one of the above radiologic signs were present based on the interpretation of an expert radiologist. In the presence of multiple radiologic signs and/or lesions affecting multiple segments of the bowel, or the presence of abscess or active fistula (fistula with fluid collection in the lumen and wall enhancement), the result was defined as 'significant' or 'severe' MRI disease activity. An MRI score was not calculated. The MRI results were compared to the clinical activity scores (CDAI) and laboratory activity markers (CRP) for correlation.

Clinical outcomes were collected. Changes in medical therapy, hospitalizations and surgery requirements were also evaluated. All changes in medical therapy were registered including antibiotics, local or systemic corticosteroids, immunosuppressive agents and biologics. Escalation in medical therapy was defined as an initiation of a higher therapeutic step (immunosuppressives or biologics) or a dose intensification of current biological therapy.

\section{Statistical analysis}

Statistical analysis was performed using the SPSS software v. 20.0 (Chicago, IL). Descriptive statistics were calculated and variables were tested for normality using Shapiro-Wilk's $\mathrm{W}$-test. The $\chi^{2}$ test and $\chi^{2}$-test with Yates correction was used 
to describe associations between categorical clinical variables. For categorical data, frequency distributions were analyzed, for continuous variables, medians and interquartile ranges were calculated. The Chi-square test was used to evaluate differences within subgroups of patients. A p-value of $<0.05$ was regarded as statistically significant.

\section{Ethical statement}

The study protocol was approved by the Semmelweis University Regional and Institutional Committee of Science and Research Ethics (SE TUKEB 142/2010). The study complies with the principles of the Declaration of Helsinki.

\section{RESULTS}

A total of 90 fast-track MRIs were performed in 75 referral CD patients (male/female: 51/49, median age: 34, IQR: 25-43 years). Location of CD was ileo-colonic in $61 \%$, colonic in $28 \%$, with perianal fistulas in $56 \%$ of patients. Previous surgery was recorded in $55 \%$ of the patients. Patient characteristics are detailed in Table I.

Magnetic resonance imaging identified luminal activity, fistula, abscess and stenosis in $44.4 \%, 35.6 \%, 22.2 \%$ and $13.3 \%$ of the scans, respectively. The MRI performed on patients

Table I. Patient characteristics (75 referral patients, 90 MRI scans)

\begin{tabular}{ll}
\hline Male/Female n (\%) & $38 / 37(51 / 49)$ \\
Median age (IQR), years & $34(25-43)$ \\
Location (L1/L2/L3/L4) (\%) & $8.0 / 28.0 / 61.3 / 2.7$ \\
Behaviour (B1/B2/B3/perianal) (\%) & $28.0 / 25.3 / 45.3 / 56.0$ \\
EIM (\%) & 42.7 \\
Median time to MRI (IQR), years & $8.8(4-18)$ \\
Colonoscopy ${ }^{n}$ (\%) & $28(31.1)$ \\
US/CT* n (\%) & $11 / 6(12.2 / 6.6)$ \\
$\begin{array}{l}\text { Type of MRI examination (abdominal-pelvic } \\
\text { MRE / abdominal MRI / pelvic MRI), n }\end{array}$ & $75 / 8 / 7$ \\
$\begin{array}{l}\text { Patients in clinical remission at time of MRI } \\
\text { (CDAI<150, or no drainig fistulas), n (\%) }\end{array}$ & $53(58.9)$ \\
$\begin{array}{l}\text { Patients in biochemical remission at time of MRI } \\
\text { (CRP < 10mg/L), n (\%) }\end{array}$ & $50(55.6)$ \\
$\begin{array}{l}\text { Treatment at time of MRI (no IS or biologics/ } \\
\text { aTNF/ IS/ aTNF+IS) (\%) }\end{array}$ & $13.3 / 22.2 / 25.6 / 38.9$ \\
Previous surgery, n (\%) & $41(54.7)$ \\
\hline
\end{tabular}

${ }^{*}$ within 3 months after MRI; n: number; EIM: extraintestinal manifestations; IS: immunosuppressives. with clinical or biomarker activity identified intestinal as well as extraintestinal findings in a much larger proportion than in patients with clinical and biomarker remission (Table II.). Fast-track MRI was followed by a change in the medical therapy (including initiation of antibiotics, corticosteroids, immunosuppressive agents and biologics) in $50.0 \%$ of all patients, with a surgery and hospitalization rates of both $21.1 \%$.

The value of fast-track MRI in patients with clinically active disease

The indication for fast-track MRI examination was active disease (clinical or biomarker activity) in $55.6 \%(n=50)$ of the patients. The MRI identified radiological activity (including mild radiological signs to severe lesions) in $94 \%$ and significant MRI activity (severe radiological lesions) in $68 \%(n=34)$ of these patients (Table II).

The MRI resulted in a therapeutic strategy change in $80 \%$ of the patients with clinical or biomarker activity $(94.1 \%$ vs. $50 \%$ in patients with significant/severe vs. no severe MRI radiological activity; $\mathrm{p}=0.001$ ). The therapeutic step was accelerated (initiation of immunosuppressives or biologics) and/or current biologic therapy was dose-optimized or switched to another biologic in a greater proportion of patients with significant MRI activity compared to patients with only a clinical/biomarker but no severe MRI activity (52.9\% vs. $18.8 \% ; \mathrm{p}=0.022$ ).

Thirty-eight percent of the patients with clinical and/or biomarker activity required surgery within three months after the MRI scan. Significant MRI radiological activity resulted in significantly higher surgery rates compared to patients without MRI radiological activity ( $50 \%$ vs. $12.5 \%, \mathrm{p}=0.013$ ). Hospitalization rates were altogether $36 \%$, higher in patients with severe MRI activity compared with patients without MRI activity (44.1\% vs. $18.8 \%$; $\mathrm{p}=0.057)$. Change in therapeutic strategy, surgery and hospitalization rates following the MRI scans are shown in detail in Fig. 1.

In a sensitivity analysis, in clinically active patients with known perianal manifestation $(n=34)$, therapeutic strategy change $(92.9 \%$ vs. $50.0 \%, \mathrm{p}=0.029)$, initiation of antibiotics (78.6\% vs. $16.7 \%, \mathrm{p}=0.008)$ and surgery $(50.0 \%$ vs. $0.0 \%$, $\mathrm{p}=0.031)$ rates were significantly more frequent following severe radiological findings on MRI (active fistula or abscess) compared to patients with no severe radiological activity. Similarly, in the subgroup of patients with B2, B3 phenotype and/or perianal manifestation $(n=43)$, change in therapeutic strategy ( 93.8 vs. $54.5 \%, \mathrm{p}=0.008$ ), initiation of antibiotics

Table II. MRI findings in patients with disease activity/flare and patients with remission

\begin{tabular}{|c|c|c|c|c|}
\hline & $\begin{array}{l}\text { All MRI scans } \\
\quad(\mathrm{n}=90)\end{array}$ & $\begin{array}{l}\text { In pts with clinical or } \\
\text { biomarker activity } \\
\qquad(\mathrm{n}=50)\end{array}$ & $\begin{array}{l}\text { In pts with clinical and } \\
\text { biomarker remission** } \\
\qquad(\mathrm{n}=40)\end{array}$ & $\mathrm{P}$ value \\
\hline Radiological activity (\%) & 68.9 & 94.0 & 37.5 & $<0.001$ \\
\hline Severe radiological activity (\%) & 40.0 & 68.0 & 5.0 & $<0.001$ \\
\hline Luminal activity (\%) & 43.3 & 58.0 & 25.0 & 0.002 \\
\hline Fistula (\%) & 35.6 & 54.0 & 12.5 & 0.001 \\
\hline Abscess (\%) & 22.2 & 38.8 & 2.5 & 0.001 \\
\hline Stenosis (\%) & 12.2 & 18.0 & 5.0 & 0.058 \\
\hline
\end{tabular}


(75.0\% vs. $27.3 \%, \mathrm{p}=0.01$ ), therapeutic step-up or optimization of current biologic treatment $(50.0 \%$ vs. $18.2 \%, \mathrm{p}=0.086)$, surgery $(53.1 \%$ vs. $9.1 \%, \mathrm{p}=0.014)$ and hospitalization $(43.8 \%$ vs. $9.1 \%, \mathrm{p}=0.037$ ) were more frequent in patients with severe radiological findings on MRI compared to patients with no severe radiological activity.

MRI results in patients with no clinical or biomarker disease activity

Significant MRI activity was detected only in $5.0 \%$ of patients in clinical and biomarker remission, while any radiological sign of active disease was confirmed in $37.5 \%$ $(n=15)$ of these patients (mainly discrete activity signs).

Change in medical therapy was recorded in $20.0 \%$ of patients with any radiological activity on MRI, compared to $8.0 \%$ in patients with no signs of radiological disease activity $(\mathrm{p}=0.098)$. Medical therapy was escalated in two patients based on MRI activity and in one patient current biologic therapy was deescalated based on the lack of active disease on MRI.

No surgery was required in patients with clinical and biomarker remission based on the MRI result, and only one patient required hospitalization. Changes in therapeutic strategy, surgery and hospitalization rates are shown in detail in Fig. 2.

Correlation between MRI radiological activity, clinical disease activity and biomarkers

Agreement between clinical activity and biomarker positivity $(\mathrm{CRP}>10 \mathrm{mg} / \mathrm{L})$ vs. significant MRI radiological activity was moderate (kappa: 0.654 and 0.591 ). The agreement was almost the same comparing patients with either clinical or biomarker positivity or both clinical and biomarker positivity vs. MRI (kappa: 0.609 and 0.638).

Colonoscopy within three months after MRI assessment was available in 28 patients, while US or CT in 17 patients. Agreement between luminal active disease on MRI and colonoscopy was moderate (kappa: 0.652). Overall agreement between luminal active disease on MRI and other imaging methods (US or CT) assessing luminal activity was good (kappa: 0.717); however, the sample size was small.

\section{DISCUSSION}

The present study evaluated the impact of fast-track MRI on patient management and clinical decision making in a specialized tertiary referral IBD center. The addition of fasttrack MRI significantly contributed to clinical decision making in CD patients with clinical or biomarker activity, leading to better patient stratification and earlier optimization of the therapeutic strategy (medical or surgical). In addition, the routine use of MRI is not indicated in patients in clinical and biomarker remission, since the proportion of patients with significant novel findings is low.

The therapeutic goals and monitoring in IBD have significantly changed in the last decade with the recent consensus (STRIDE) [2], suggesting that disease activity should be monitored objectively by endoscopy and the clinical
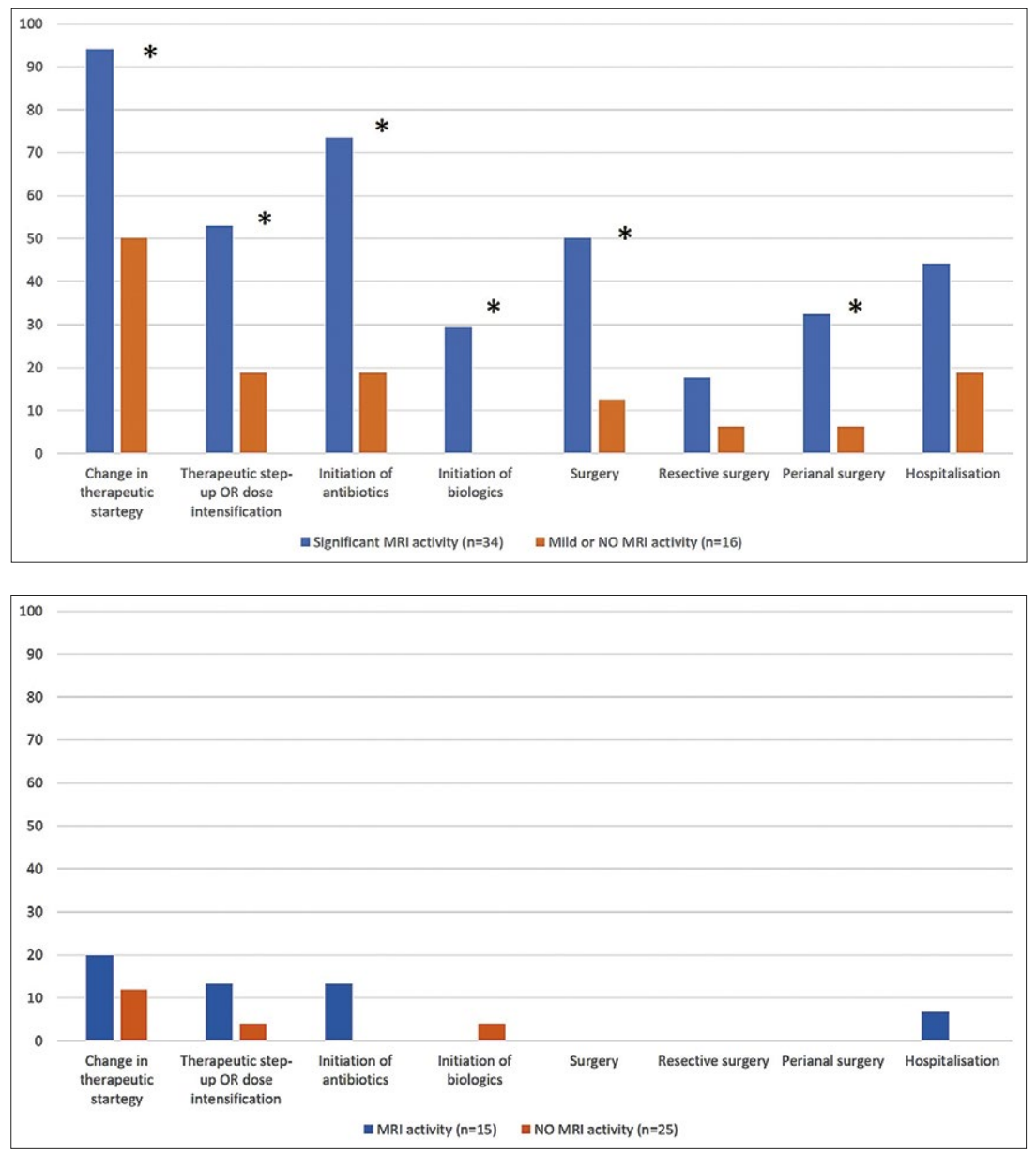

Fig. 1. Change in therapeutic strategy, surgery and hospitalization rates following the MRI scans in patients with clinical or biomarker activity.
Fig. 2. Change in therapeutic strategy, surgery and hospitalization rates following the MRI scans in patients without clinical or biomarker activity 
parameters with biomarkers as adjunct measures. Of note, our center has applied objective patient evaluation and follow-up policy including open clinic concept and fast-track access to specialist consultation, endoscopy and imaging [11]. However, it is still questionable if routine cross-sectional imaging can be regarded as a valid alternative for endoscopy in all IBD patients.

Recently, multiple studies have reported that MRI/MRE can be used effectively as a non-invasive, cross-sectional imaging method for the diagnosis of IBD, and that it is able to identify also extraluminal complications $[6,12]$. Comparative studies and meta-analyses summarized that sensitivity estimates for the diagnosis of IBD were high and not significantly different between MRI and CT and abdominal US imaging modalities $[3,5,6,13-17]$. In addition, in CD up-to-date MRI/MRE imaging modalities were reported to have a good agreement with endoscopic activity [17]. Rimola et al. [18] reported their results from a cross-sectional study comprising 50 patients, with the aim of characterizing the MRI patterns of the terminal ileum and the colon in patients with active and inactive CD to establish the MR changes indicative of mild and severe intestinal lesions. The comparison of intestinal segments with absent, mild and severe inflammation on endoscopy demonstrated a progressive and significant $(\mathrm{p}<0.001)$ increase in a set of MRI parameters, including wall thickness, postcontrast wall signal intensity, presence of oedema, relative contrast enhancement, ulcers, pseudopolyps and lymph node enlargement. Independent predictors for Crohn's Disease Endoscopic Index of Severity (CDEIS) were wall thickness $(p=0.007)$, relative contrast enhancement $(p=0.01)$, presence of oedema $(p=0.02)$ and presence of ulcers at MRI scans $(\mathrm{p}=0.003)$. The authors concluded that MRI can be considered as an alternative to colonoscopy for the assessment of ileocolonic CD and can be complementary to endoscopy in evaluating disease activity in other bowel segments. Good concordance between MRI and endoscopic findings has been also reported by other studies, in parallel with results from the present study [19-22].

Multiple groups have developed MRI-based severity scores in patients with CD $[18,23,24]$. The Spanish IBD study group developed and validated the Magnetic Resonance Index of Activity (MaRIA) to assess disease severity in CD. The score includes wall thickness, relative contrast enhancement, and the presence of oedema and ulcers as identified on MRI. They reported a good correlation between the presence and severity of endoscopic lesions and MRE ( $\mathrm{r}=0.83, \mathrm{p}<0.001)$ [23, 25]. Another validated score system is a modified version of the MaRIA score ('Clermont score') for the assessment of ileal inflammatory activity using DWI MRI sequences with the benefit of avoiding gadolinium contrast injection [21].

Comparison of MRI with other diagnostic tools measuring disease activity was also reported. A significant correlation of the global MaRAI score with Harvey-Bradshaw index (HBI) $(\mathrm{r}=0.56, \mathrm{p}<0.001)$ and $\mathrm{CRP}(\mathrm{r}=0.53, \mathrm{p}<0.001)$ were reported $[18,24]$. However, a later study of the same cohort performing the validation of MaRAI score on disease follow-up found a low correlation between MaRAI and CDAI scores at initial patient evaluation $(\mathrm{r}=0.14 ; \mathrm{p}=0.32)$ [25]. These results are not surprising as the potentially high variability between clinical, biomarker and endoscopic activity is well-known [26, 27].
Studies have reported also that MRI/MRE results can significantly impact therapeutic decisions. In concordance with the findings of the present study, a study from the Spanish group demonstrated that MRE had superior diagnostic accuracy compared to ileocolonoscopy in stricturing and penetrating disease, and had a higher impact on therapeutic decisions (starting anti-TNF therapy or surgery) in patients with moderate or severe CD [7]. Authors suggested that MRI might be considered the method of choice, particularly in centers with experienced radiologists. A Japanese study also suggested that MRI was superior to endoscopy in detecting intestinal fibrosis and might identify more accurately patients who would respond to anti-inflammatory therapy $[28,29]$. In summary, MRI had a comparable accuracy to endoscopic evaluation supporting its routine use in the clinical practice if the necessary expertise and access is available [30].

Similar to the findings of the present study, a previous small prospective study from Ireland in patients with smallbowel CD reported that MRE had a large impact on patient management. The majority of patients who underwent MRE had a change in therapeutic management (74\%), in those with an abnormal MRE this percentage was even higher (82\%). After MRE, $47 \%$ of patients had surgery and $53 \%$ had changes in medical treatment [31]. However, an evaluation based on the combination of MRI and biomarkers with or without clinical symptoms might be more accurate and might better reflect the everyday clinical practice as suggested also by the present study. In our study, we analyzed the impact of MRI separately in patients with or without clinical or biomarker activity/positivity. Among patients with associated clinical disease activity, a change in medical therapy or surgery was indicated in a significantly greater proportion if significant MRI activity was also present. These data confirm the need for objective assessment in patients with clinical and biomarker activity including cross sectional imaging evaluation and highlight the additive role of fast-track MRI on patient management in this setting. Similarly, Klang et al. [32] showed that restricted diffusion on MRI in combination with elevated fecal calprotectin level is a good predictor for active inflammation. Their results suggest that the DWI MRI technique could be a good clinical follow-up method to identify subclinical inflammation in patients with a high risk for complications.

The strengths of the present study include the commitment to objective patient monitoring practices in our IBD center. Fast-track MRI was initiated as part of this objective patientmonitoring algorithm, in addition to multidisciplinary meetings with surgeons and radiologists. All MRE scans were performed and reviewed by the same expert radiologist with a high experience in IBD radiology. A limitation of our study is that formal MRI scores were not calculated. Furthermore, being a retrospective study, comparative assessment of clinical decisions with and without MRI was not available.

\section{CONCLUSIONS}

Fast-track MRI had a significant impact on patient management and clinical decision making in our CD patients with clinical and biomarker activity, leading to better patient 
stratification and earlier optimization of the therapy (medical or surgical). If expertise and access is available, MRI is the cross-sectional method of choice in CD patients with extensive or complicated disease (including those with perianal complication), since it has no radiation burden and may identify intestinal activity as well as extraintestinal findings. It also helps to avoid unnecessary endoscopies, thus decreasing the procedure burden for the patients. In contrast, MRI reveals significant novel finding only in a small proportion of patients without clinical and biomarker activity, thus its routine use in this patient subset is not indicated. The present study advocates for the use of fast-track MRI as part of the objective patient management algorithm in CD patients with clinical and biomarker disease activity to enable a more accurate patient characterization and decision-making.

Conflicts of interest: None to declare.

Authors' contributions: A.I. and B.D.L. performed the data collection and drafted the manuscript. L.G., Z.K., Z.V. and P.A.G. performed the data collection. G.R. reviewed the MRI scans as an expert radiologist with high experience in IBD. P.L.L. conceived the study and consulted the concept, performed data collection and validation, carried out the statistical analysis, supervised the manuscript preparation and is acting as the submission's guarantor. All authors read and approved the final manuscript.

\section{REFERENCES}

1. Peyrin-Biroulet L, Loftus EV Jr, Colombel JF, Sandborn WJ. Longterm complications, extraintestinal manifestations, and mortality in adult Crohn's disease in population-based cohorts. Inflamm Bowel Dis 2011;17:471-478. doi:10.1002/ibd.21417

2. Peyrin-Biroulet L, Sandborn W, Sands BE, et al. Selecting Therapeutic Targets in Inflammatory Bowel Disease (STRIDE): Determining Therapeutic Goals for Treat-to-Target. Am J Gastroenterol 2015;110:1324-1338. doi:10.1038/ajg.2015.233

3. Gomollón F, Dignass A, Annese V, et al. 3rd European Evidence-based Consensus on the Diagnosis and Management of Crohn's Disease 2016: Part 1: Diagnosis and Medical Management. J Crohns Colitis 2017;11:325. doi:10.1093/ecco-jcc/jjw168

4. Ordás I, Rimola J, Rodriguez S, et al. Accuracy of magnetic resonance enterography in assessing response to therapy and mucosal healing in patients with Crohn's disease. Gastroenterology 2014;146:374-382. doi:10.1053/j.gastro.2013.10.055

5. Panes J, Bouhnik Y, Reinisch W, et al. Imaging techniques for assessment of inflammatory bowel disease: joint ECCO and ESGAR evidence-based consensus guidelines. J Crohns Colitis 2013;7:556-585. doi:10.1016/j. crohns.2013.02.020

6. García-Bosch O, Ordás I, Aceituno M, et al. Comparison of Diagnostic Accuracy and Impact of Magnetic Resonance Imaging and Colonoscopy for the Management of Crohn's Disease. J Crohns Colitis 2016;10:663669. doi:10.1093/ecco-jcc/jjw015

7. Panes J, Bouzas R, Chaparro M, et al. Systematic review: the use of ultrasonography, computed tomography and magnetic resonance imaging for the diagnosis, assessment of activity and abdominal complications of Crohn's disease. Aliment Pharmacol Ther 2011;34:125145. doi:10.1111/j.1365-2036.2011.04710.x
8. Novak K, Tanyingoh D, Petersen F, et al. Clinic-based Point of Care Transabdominal Ultrasound for Monitoring Crohn's Disease: Impact on Clinical Decision Making. J Crohns Colitis 2015;9:795-801. doi:10.1093/ ecco-jcc/jjv105

9. Strohl M, Gonczi L, Kurt Z, Bessissow T, Lakatos PL. Quality of care in inflammatory bowel diseases: What is the best way to better outcomes? World J Gastroenterol 2018;24:2363-2372. doi:10.3748/wjg.v24.i22.2363

10. Best WR, Becktel JM, Singleton JW. Rederived values of the eight coefficients of the Crohn's disease activity index [CDAI]. Gastroenterology 1979;77:843-846.

11. Gonczi L, Kurti Z, Golovics PA, et al. Quality of care indicators in inflammatory bowel disease in a tertiary referral center with open access and objective assessment policies. Dig Liver Dis 2018;50:37-41. doi:10.1016/j.dld.2017.09.137

12. Lee SS, Kim AY, Yang SK, et al. Crohn disease of the small bowel: comparison of CT enterography, MR enterography, and small-bowel follow-through as diagnostic techniques. Radiology 2009;251:751-761. doi:10.1148/radiol.2513081184

13. Qiu Y, Mao R, Chen BL, et al. Systematic review with meta-analysis: magnetic resonance enterography vs. computed tomography enterography for evaluating disease activity in small bowel Crohn's disease. Aliment Pharmacol Ther 2014;40:134-146. doi:10.111// apt.12815

14. Siddiki HA, Fidler JL, Fletcher JG, et al. Prospective comparison of state-of-the-art MR enterography and CT enterography in small-bowel Crohn's disease. AJR Am J Roentgenol 2009;193:113-121. doi:10.2214/ AJR.08.2027

15. Horsthuis K, Bipat S, Bennink RJ, Stoker J. Inflammatory bowel disease diagnosed with US, MR, scintigraphy, and CT: meta-analysis of prospective studies. Radiology 2008;247:64-79. doi:10.1148/ radiol.2471070611

16. Fiorino G, Bonifacio C, Peyrin-Biroulet L, et al. Prospective comparison of computed tomography enterography and magnetic resonance enterography for assessment of disease activity and complications in ileocolonic Crohn's disease. Inflamm Bowel Dis 2011;17:1073-1080. doi:10.1002/ibd.21533

17. Hordonneau C, Buisson A, Scanzi J, et al. Diffusion-weighted magnetic resonance imaging in ileocolonic Crohn's disease: validation of quantitative index of activity. Am J Gastroenterol 2014;109:89-98. doi:10.1038/ajg.2013.385

18. Rimola J, Rodriguez S, García-Bosch O, et al. Magnetic resonance for assessment of disease activity and severity in ileocolonic Crohn's disease. Gut 2009;58:1113-1120. doi:10.1136/gut.2008.167957

19. Dinter DJ, Chakraborty A, Brade J, et al. Endoscopy and magnetic resonance imaging in patients with Crohn's disease: a retrospective single-centre comparative study. Scand J Gastroenterol 2008;43:207216. doi:10.1080/00365520701676021

21. Grand DJ, Kampalath V, Harris A, et al. MR enterography correlates highly with colonoscopy and histology for both distal ileal and colonic Crohn's disease in 310 patients. Eur J Radiol 2012;81:e763-e739. doi:10.1016/j.ejrad.2012.02.011

22. Oussalah A, Laurent V, Bruot O, et al. Diffusion-weighted magnetic resonance without bowel preparation fordetecting colonic inflammation in inflammatory bowel disease. Gut 2010;59:1056-1065. doi:10.1136/ gut.2009.197665

22. Rajabi S, Dupuis I, Benmassaoud A, et al. Magnetic Resonance Enterography in the Study of Patients With Crohn's Disease: Which Findings Are More Likely to Change Patient Management? Can Assoc Radiol J 2016;67:387-394. doi:10.1016/j.carj.2016.02.004 
23. Makanyanga JC, Pendsé D, Dikaios N, et al. Evaluation of Crohn's disease activity: initial validation of a magnetic resonance enterography global score (MEGS) against faecal calprotectin. Eur Radiol 2014;24:277-287. doi:10.1007/s00330-013-3010-z

24. Rimola J, Ordás I, Rodriguez S, et al. Magnetic resonance imaging for evaluation of Crohn's disease: validation of parameters of severity and quantitative index of activity. Inflamm Bowel Dis 2011;17:1759-1768. doi:10.1002/ibd.21551

25. Ordás I, Rimola J, Rodríguez S, et al. Accuracy of magnetic resonance enterography in assessing response to therapy and mucosal healing in patients with Crohn's disease. Gastroenterology 2014;146:374-382.e1. doi:10.1053/j.gastro.2013.10.055

26. Jones J, Loftus EV Jr, Panaccione R, et al. Relationships between disease activity and serum and fecal biomarkers in patients with Crohn's disease. Clin Gastroenterol Hepatol 2008;6:1218-1241. doi:10.1016/j.cgh.2008.06.010

27. Schoepfer AM, Beglinger C, Straumann A, et al. Fecal calprotectin correlates more closely with the Simple Endoscopic Score for Crohn's disease (SES-CD) than CRP, blood leukocytes, and the CDAI. Am J Gastroenterol 2010;105:162-169. doi:10.1038/ajg.2009.545
28. Higgins PD. Measurement of fibrosis in Crohn's Disease strictures with imaging and blood markers to inform clinical decisions. Dig Dis 2017;35:32-37. doi:10.1159/000449080

29. Naganuma M, Okuda S, Hisamatsu T, et al. Findings of ulceration and severe stricture on MRE can predict prognosis of Crohn's disease in patients treated with anti-TNF treatment. Abdom Radiol 2017;42:141151. doi:10.1007/s00261-016-0878-5

30. Buisson A, Pereira B, Goutte M, et al. Magnetic resonance index of activity (MaRIA) and Clermont score are highly and equally effective MRI indices in detecting mucosal healing in Crohn's disease. Dig Liver Dis 2017;49:1211-1217. doi:10.1016/j.dld.2017.08.033

31. Cheriyan DG, Slattery E, McDermott S, et al. Impact of magnetic resonance enterography in the management of small bowel Crohn's disease. Eur J Gastroenterol Hepatol 2013;25:550-555. doi:10.1097/ MEG.0b013e32835d4e9c

32. Klang E, Kopylov U, Eliakim R, et al. Diffusion-weighted imaging in quiescent Crohn's disease: correlation with inflammatory biomarkers and video capsule endoscopy. Clin Radiol 2017;72:798.e7-798.e13. doi:10.1016/j.crad.2017.04.006 